\title{
Marketing Policy that Accelerate Tobacco Use in Bangladesh: A Statistical Investigation
}

Papia Sultana ${ }^{1 *}$, Tahidur Rahman $\mathbf{M}^{1,2}$ and Dulal Chandra Roy ${ }^{1}$

${ }^{1}$ Department of Statistics, University of Rajshahi, Bangladesh

${ }^{2}$ Safi Consultancy, Sylhet, Bangladesh

\section{Abstract}

Background: Tobacco use is a manmade manner which causes severe chronic diseases and Bangladesh is one of the most tobacco prevalent countries in the world. Advertisement and promotion events may have a big contribution to accelerate this. Therefore, this study aimed to analyze the advertisement and promotion events that encouraged the tobacco user.

Data and methods: Secondary data of sample size 9629 collected by the Global Adult Tobacco Survey (GATS), 2010 has been used. Along with descriptive analysis, binary logistic regression has been used to analyze the sociodemographic and economic correlates to be encouraged by marketing policy.

Results: The most common site for noticing cigarette, bidi and smokeless tobacco product advertisements was in stores $(49.90 \%, 26.25 \%$ and $13.97 \%)$. From logistic regression it has been found that rural respondents are 1.17 times more inspired to smoke $(\mathrm{OR}=1.17,95 \% \mathrm{Cl}=1.06,1.30)$ from marketing policy than urban respondents. Female respondents are less inspired to smoke $(\mathrm{OR}=0.24,95 \% \mathrm{Cl}=0.20,0.28)$ than male respondents. Older respondents are less inspired to smoke by marketing policy than younger respondents $(\mathrm{OR}=0.98,95 \% \mathrm{Cl}=0.98,0.99)$. On the other hand, Rural respondents are 1.15 times more likely to be inspired to use smokeless product than urban respondents $(\mathrm{OR}=1.15,95 \% \mathrm{Cl}=1.02,1.31)$. Female respondents are 0.63 times less inspired to use smokeless tobacco product than male respondents $(\mathrm{OR}=0.63,95 \% \mathrm{Cl}=0.51,0.77)$ by marketing policy. Older respondents are less inspired to use smokeless tobacco products by marketing policy than younger respondents $(\mathrm{OR}=0.99,95 \% \mathrm{Cl}=0.98,0.99)$.

Conclusion: To reduce tobacco use in Bangladesh, Government, policy makers and research institutions that are working for reduction of tobacco use should pay attention more on young, student and female to advocate more. Also, Government could take action to limit advertisement in selling store.

Keywords: Marketing policy; Tobacco use; Logistic regression; Odds ratio, GATS

\section{Introduction}

Smoking is a manmade manner which causes chronic diseases, such as ischemic heart disease, cerebrovascular disease, pulmonary obstructive disease and cancers [1]. One-third to one-half of tobacco users die from the effects of tobacco smoking. Smoking causes 1 out of 10 deaths among adult's worldwide [2]. Many socio-economic factors are associated with smoking [3,4]. The shift of the tobacco epidemic to the developing world will lead to unprecedented levels of disease and early death in countries where population growth and the potential for increased tobacco smoking are highest and where health-care services are least available [5]. Smokeless tobacco consumption (STC) is as dangerous as tobacco smoking which causes oral, head and neck cancer, diabetes, hypercholesterolemia, myocardial infarction and adverse effects on pregnancy [6].

Bangladesh has a long history of tobacco use and a variety of ways in which tobacco is smoked and smokeless tobacco is used. Previously, tobacco was a less priority issue as research topic in Bangladesh. After realization of adverse effect of tobacco consumption, Government, non-government organizations and research institutions are paying attention to it. But, still few papers have been found in literature related to tobacco in Bangladesh. Most of them are related to pattern and prevalence [7-12]. Few papers are related to economic issues of tobacco use Nargis, et al. [13]; Barkat, et al. [14] and knowledge and awareness [15]. This is the first study that aimed to find out the marketing policies in Bangladesh which encouraged the users in the last 30 days and hence playing role against reduction of tobacco use from a nationwide survey.
This study also aimed to identify significant characteristics of the group of people who were encouraged by such marketing policy.

\section{Data and Methodology}

Secondary data collected by the Global Adult Tobacco Survey (GATS), 2010 (http://www.who.int/tobacco/surveillance/survey/gats/ en/) has been used in this paper. In Bangladesh, GATS was conducted in 2009 as a household survey of persons 15 years of age or older by the National Institute of Preventive and Social Medicine in collaboration with the Bangladesh Bureau of Statistics (BBS) and National Institute of Population Research and Training (NIPRT). There were a total of 9629 completed interviews (male $=4468$ and female $=5161$ ). Details about the survey methods, questionnaire, and definitions of various terminologies can be found [16,17].

The wealth index was constructed by the GATS Collaborator Team using principal component analysis (PCA) method. Asset information covered household ownership of a number of items, such

${ }^{*}$ Corresponding author: Papia Sultana, Department of Statistics, University of Rajshahi, Bangladesh, Tel: +880 721-750244; E-mail: papia.stat@ymail.com

Received November 07, 2017; Accepted December 15, 2017; Published December 29, 2017

Citation: Sultana P, Rahman MT, Roy DC (2017) Marketing Policy that Accelerate Tobacco Use in Bangladesh: A Statistical Investigation. J Biom Biostat 8: 386. doi: 10.4172/2155-6180.1000386

Copyright: $\odot 2017$ Sultana $P$, et al. This is an open-access article distributed unde the terms of the Creative Commons Attribution License, which permits unrestricted use, distribution, and reproduction in any medium, provided the original author and source are credited. 
as electricity, flush toilet, fixed telephone, cell telephone, television, radio, refrigerator, car, moped/scooter/motorcycle, washing machine, bicycle, sewing machine, almirah/ wardrobe, table, bed or cot, chair or bench, watch or clock, as well as the type of main material used for the roof of the main house (cement, tin and katcha such as bamboo/ thatched/straw).

Marketing policy for cigarette or bidi includes (i) advertisements (ii) signs promotion of cigarette or bidi and (iii) sponsorship of cigarette or Bidi Company in sports or sporting events in the preceding 30 days that inspired the user(s). Marketing policy for cigarette or bidi in stores (where cigarettes or bidi are sold) includes (i) cigarettes or bidi at sale prices, (ii) free gifts, or (iii) discount offers on other products while buying cigarettes or bidi, (iv) any advertisements or signs promoting cigarettes or bidi in the preceding 30 days that encouraged the user(s). Marketing policy for cigarette or bidi in places other than stores (where cigarettes or bidi are sold) includes (i) advertisements or signs promoting cigarettes or bidi and (ii) sponsorship of cigarette or bidi company in sporting events in the preceding 30 days that inspired the users (Figure 1).

Marketing policy for smokeless tobacco product includes (i) advertisements (ii) signs promotion of smokeless tobacco and (iii) sponsorship of smokeless tobacco Company in sports or sporting events in the preceding 30days that inspired the users. Marketing policy for smokeless tobacco in stores (where smokeless tobacco products are sold) includes (i) smokeless tobacco at sale prices, (ii) free gifts, or (iii) discount offers on other products while buying smokeless tobacco (iv) any advertisements or signs promoting smokeless tobacco in the preceding 30 days that inspired the users. Marketing policy for smokeless tobacco in places other than stores (where smokeless tobacco are sold) includes (i) advertisements or signs promoting smokeless tobacco and (ii) sponsorship of smokeless tobacco company in sporting events in the preceding 30 days that inspired the users.

Among various statistical methods, descriptive analysis has been performed to know the characteristics of the study subjects. For that frequency with percentage has been reported for categorical data and mean with standard deviation has been reported for continuous data. Comparisons of marketing policy to confounding variables (residence and gender) have been performed, too. To compare variables chisquare test (Pearson Chi-square or Likelihood Ratio Chi-square) has been used and prevalence with 95\% confidence interval has been reported; various socio-demographic and economic characteristics have been compared to marketing policy, too. On the other hand, t-test to compare mean Rao [18] has been used for continuous data

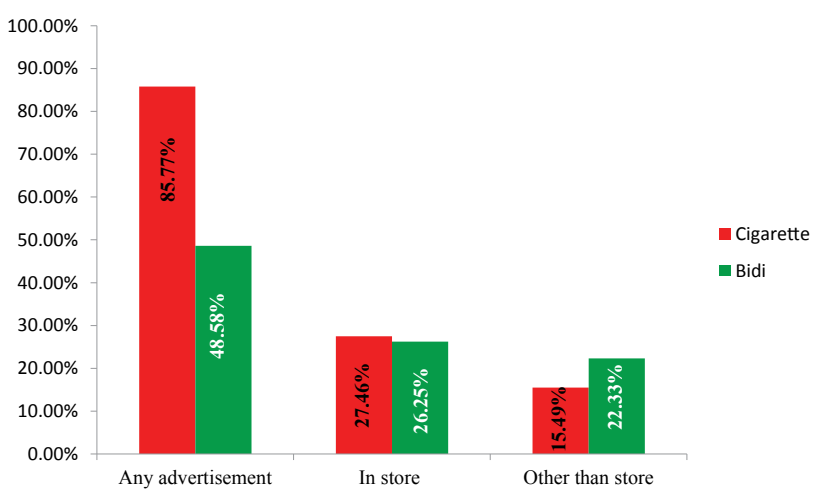

Figure 1: Marketing policy (tobacco smoking products).

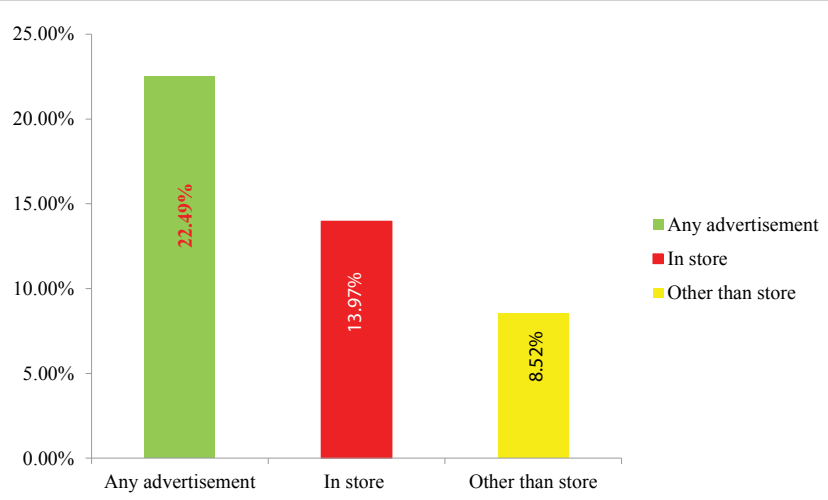

Figure 2: Marketing policy (smokeless tobacco products)

and mean with standard deviation has been reported. All the tests have been performed at $5 \%$ level of significance (Figure 2). To identify the significant socio-demographic and economic correlates to marketing policy that inspired the user in the preceding 30 days, binary logistic regression has been used and Odds Ratio (OR) with 95\% confidence interval has been reported [19]. Statistical software StataSE version 11 (StataCorp, USA) has been used to carry out statistical analyses.

\section{Results}

Characteristic of study subject has been reported in Table 1 . The percentage of people aged 15 years or above who noticed some cigarette advertisement in anywhere, in last 30 days that encouraged the tobacco users was $85.77 \%$ (Tables 2-6). The most common site for noticing cigarette advertisements was in a store (49.90\%) and other than store was $35.87 \%$. Sport promotional event, in last 30 days that encouraged the tobacco users was $2.07 \%$ and other than promotional event were $31.00 \%$.

The percentage of people who noticed some bidi advertisement in anywhere was $48.58 \%$. The most common site for noticing such advertisement was in stores $(26.25 \%)$ and other than store were $22.33 \%$. Sport promotional event were $0.65 \%$ and other than promotional event were $13.14 \%$.

The percentage of people who noticed some smokeless tobacco product advertisement was $22.49 \%$. The most common site was in a store $(13.97 \%)$ and other than store was $(8.52 \%)$. Sport promotional event was $0.21 \%$ and other than promotional event was $4.02 \%$.

From logistic regression (Table 7), it has been found that current tobacco smokers are 1.25 times more likely to be inspired by the marketing policy in preceding 30 days to smoke than their counter parts $(\mathrm{OR}=1.25,95 \% \mathrm{CI}=1.10,1.42)$ and rural respondents are 1.17 times more inspired to smoke $(\mathrm{OR}=1.17,95 \% \mathrm{CI}=1.06,1.30)$ from marketing policy than urban respondents. Female respondents are less inspired to smoke $(\mathrm{OR}=0.24,95 \% \mathrm{CI}=0.20,0.28)$ than male respondents. Older respondents are less inspired to smoke by marketing policy than younger respondents ( $\mathrm{OR}=0.98,95 \% \mathrm{CI}=0.98,0.99)$. Respondents who has completed high school level of education $(\mathrm{OR}=1.49,95 \% \mathrm{CI}=1.17$, $1.90)$ and College/University $(\mathrm{OR}=1.65,95 \% \mathrm{CI}=1.28,2.11)$ are more likely to be inspired by the marketing policy to smoke. It has been also found that people who are doing business (employee: $\mathrm{OR}=1.55,95 \%$ $\mathrm{CI}=1.27,1.90$ ), agricultural/industrial worker/daily laborer/other selfemployed $(\mathrm{OR}=1.44,95 \% \mathrm{CI}=1.19,1.76)$ and students $(\mathrm{OR}=1.61,95 \%$ $\mathrm{CI}=1.30,2.00)$ are more likely to be inspired by the marketing policy to smoke. 
Citation: Sultana P, Rahman MT, Roy DC (2017) Marketing Policy that Accelerate Tobacco Use in Bangladesh: A Statistical Investigation. J Biom Biostat 8: 386. doi: 10.4172/2155-6180.1000386

Page 3 of 8

\begin{tabular}{|c|c|c|c|}
\hline Characteristics & Total (N=9629) Frequency (\%) & $\begin{array}{l}\text { Tobacco Smoker }(\mathrm{N}=2038) \\
\text { Frequency }(\%)\end{array}$ & $\begin{array}{l}\text { Smokeless tobacco user } \\
(\mathrm{N}=2336) \text { Frequency }(\%)\end{array}$ \\
\hline \multicolumn{4}{|l|}{ Gender } \\
\hline Male & $4468(46.40)$ & $1972(96.76)$ & $980(41.95)$ \\
\hline Female & $5161(53.60)$ & $66(3.24)$ & $1356(58.05)$ \\
\hline \multicolumn{4}{|l|}{ Residence } \\
\hline Urban & $4857(50.44)$ & $964(47.30)$ & $978(41.87)$ \\
\hline Rural & $4772(49.56)$ & $1074(52.70)$ & $1358(58.13)$ \\
\hline Age (yrs) ${ }^{*}$ & $36.90(14.90)$ & $40.50(13.57)$ & $37.00(14.58)$ \\
\hline \multicolumn{4}{|l|}{ Educational level } \\
\hline No formal schooling & $3430(35.62)$ & $958(47.01)$ & $101(47.33)$ \\
\hline Less than primary school completed & $1487(15.44)$ & $372(18.25)$ & $378(16.25)$ \\
\hline Primary School Completed & $1115(11.58)$ & $184(9.03)$ & $259(11.13)$ \\
\hline Less than secondary school completed & $1937(20.12)$ & $315(15.46)$ & $387(16.64)$ \\
\hline Secondary school completed & $663(6.89)$ & $88(4.32)$ & $102(4.39)$ \\
\hline High school completed & $463(4.81)$ & $51(2.50)$ & $64(2.75)$ \\
\hline College/University Completed and higher & $484(5.03)$ & $70(3.43)$ & $33(1.42)$ \\
\hline Don’t Know & $50(0.52)$ & $0(0.00)$ & $2(0.09)$ \\
\hline \multicolumn{4}{|l|}{ Occupation } \\
\hline Employment (Government, Non-Government) & $961(9.98)$ & $225(11.04)$ & $128(5.50)$ \\
\hline Business (small, large) & $993(10.31)$ & $440(21.59)$ & $196(8.43)$ \\
\hline Farming (land owner and farmer) & $826(8.58)$ & $411(20.17)$ & $196(8.43)$ \\
\hline $\begin{array}{l}\text { Agricultural/Industrial worker/daily laborer/Other self- } \\
\text { employed }\end{array}$ & $1537(15.96)$ & $74(3.63)$ & $46(1.98)$ \\
\hline Homemaker/Housework & $4030(41.85)$ & $628(30.81)$ & $371(15.95)$ \\
\hline Retired and unemployed (able to work/unable to work) & $431(4.48)$ & $42(2.06)$ & $1111(47.76)$ \\
\hline Student/Other & $851(8.84)$ & $83(4.07)$ & $73(3.14)$ \\
\hline \multicolumn{4}{|l|}{ Wealth index } \\
\hline Lowest & 1866 (19.38) & $492(24.14)$ & $594(25.54)$ \\
\hline Low & $2068(21.48)$ & $516(25.32)$ & $587(25.24)$ \\
\hline Middle & 1732 (17.99) & $375(18.40)$ & $440(18.92)$ \\
\hline High & $2040(21.19)$ & $392(19.23)$ & 458 (19.69) \\
\hline Highest & $1923(19.97)$ & $263(12.90)$ & $247(10.62)$ \\
\hline
\end{tabular}

*Mean (standard deviation) has been reported.

Table 1: Characteristics of the study subjects.

\begin{tabular}{|l|c|c|}
\hline Marketing policy & Smoking (N=2038) \\
\hline Any advertisement (Cigarettes) & $1748(85.77)$ \\
\hline In store & $1017(49.90)$ \\
\hline Other than store & $731(35.87)$ \\
\hline Sport or promotion event & $29(2.07)$ \\
\hline Other promotions & $603(31.00)$ \\
\hline Any advertisement (Bidi) & $980(48.58)$ \\
\hline In store & $525(26.25)$ \\
\hline Other than store & $455(22.33)$ \\
\hline Sport or promotions event & $9(0.65)$ \\
\hline Other promotions & -- \\
\hline Any advertisement (Smokeless tobacco products) & -- \\
\hline In store & -- \\
\hline Other than store & -- \\
\hline Sport or promotion event & -- \\
\hline Other promotions & -- \\
\hline
\end{tabular}

--Indicates not applicable.

**In the last 30 days, respondent had noticed any advertisements or signs promoting events that encouraged respondent to tobacco use.

Table 2: Marketing policy that play role against tobacco use reduction in Bangladesh.

On the other hand, it has been found that smokeless tobacco users are 1.31 times more likely to be inspired by the marketing policy in preceding 30 days to use smokeless tobacco product than their counter parts $(\mathrm{OR}=1.31,95 \% \mathrm{CI}=1.14,1.51)$ (Table 7$)$. Rural respondents are 1.15 times more likely to be inspired to use smokeless product than urban respondents $(\mathrm{OR}=1.15,95 \% \mathrm{CI}=1.02,1.31)$. Female respondents are 0.63 times less inspired to use smokeless tobacco product than male respondents $(\mathrm{OR}=0.63,95 \% \mathrm{CI}=0.51,0.77)$ by marketing policy. Older respondents are less inspired to use smokeless tobacco products by marketing policy than younger respondents $(\mathrm{OR}=0.99,95 \% \mathrm{CI}=0.98$, 0.99). Respondents who has completed high school level of education $(\mathrm{OR}=1.53,95 \% \mathrm{CI}=1.14,2.05)$ and College/University $(\mathrm{OR}=1.65,95 \%$ 
Citation: Sultana P, Rahman MT, Roy DC (2017) Marketing Policy that Accelerate Tobacco Use in Bangladesh: A Statistical Investigation. J Biom Biostat 8: 386. doi: 10.4172/2155-6180.1000386

Page 4 of 8

\begin{tabular}{|c|c|c|c|c|c|c|}
\hline \multirow[t]{2}{*}{ Marketing policy ${ }^{* *}$} & \multicolumn{3}{|c|}{ Smoking } & \multicolumn{3}{|c|}{ Smokeless } \\
\hline & Male* & Female $^{*}$ & p-value & Male* & Female $^{*}$ & p-value \\
\hline Any advertisement (Cigarettes) & $98.59(97.93,99.25)$ & $1.41(0.75,2.07)$ & $<0.001$ & & & \\
\hline In store & $98.52(97.78,99.27)$ & $1.48(0.73,2.22)$ & 0.003 & & & \\
\hline Other than store & $99.31(98.71,99.92)$ & $0.68(0.08,1.29)$ & $<0.001$ & & & \\
\hline Sport or promotion event & $96.55(89.49,100.0)$ & $3.45(3.61,10.51)$ & 0.412 & & & \\
\hline Other promotions & $98.51(57.53,99.74)$ & $1.49(0.21,2.46)$ & 0.053 & & & \\
\hline Any advertisement (Bidi) & $97.83(96.75,98.92)$ & $2.16(1.07,3.24)$ & 0.067 & & & \\
\hline In store & $97.33(95.95,98.71)$ & $2.66(1.28,4.04)$ & 0.788 & & & \\
\hline Other than store & $99.12(98.25,99.98)$ & $0.88(0.018,1.75)$ & 0.002 & & & \\
\hline Sport or promotion event & $88.89(63.26,100.0)$ & $11.11(10.51,36.73)$ & 0.022 & & & \\
\hline Other promotions & $98.04(96.32,99.75)$ & $1.96(0.24,3.67)$ & 0.572 & & & \\
\hline $\begin{array}{l}\text { Any advertisement } \\
\text { (Smokeless) }\end{array}$ & & & & $55.15(49.98,60.32)$ & $44.85(39.68,50.02)$ & $<0.001$ \\
\hline In store & & & & $49.81(43.87,55.76)$ & $50.18(44.23,56.12)$ & 0.662 \\
\hline Other than store & & & & $69.34(62.88,75.80)$ & $30.65(24.19,37.11)$ & $<0.001$ \\
\hline Sport or promotion event & & & & $66.67(0.00,200.10)$ & $33.33(0.0,100)$ & 0.413 \\
\hline Other promotions & & & & $67.85(57.66,78.06)$ & $32.14(21.94,2.34)$ & $<0.001$ \\
\hline
\end{tabular}

* Proportional value with $95 \%$ confidence interval has been reported.

${ }^{*}$ In the last 30 days, respondent had noticed any advertisements or signs promoting events that encouraged respondent to tobacco use.

Table 3: Comparing various marketing policies to gender.

\begin{tabular}{|c|c|c|c|c|c|c|}
\hline \multirow[t]{2}{*}{ Marketing policies** } & \multicolumn{3}{|c|}{ Smoking } & \multicolumn{3}{|c|}{ Smokeless } \\
\hline & Urban* & Rural* $^{*}$ & p-value & Urban* & Rural $^{*}$ & p-value \\
\hline Any advertisement (Cigarettes) & $48.83(46.00,51.66)$ & $51.17(48.33,53.99)$ & 0.094 & & & \\
\hline In store & $46.60(43.53,49.67)$ & $53.39(50.32,56.46)$ & 0.420 & & & \\
\hline Other than store & $51.57(47.94,55.20)$ & $48.42(44.79,52.05)$ & 0.004 & & & \\
\hline Sport or promotion event (cigarettes) & $62.07(43.28,80.85)$ & $37.93(19.15,56.72)$ & 0.109 & & & \\
\hline Other promotions (cigarettes) & $51.24(47.24,55.24)$ & $48.76(44.75,52.75)$ & 0.096 & & & \\
\hline Any advertisement (Bidi) & $45.24(41.53,48.95)$ & $54.75(51.04,58.46)$ & 0.182 & & & \\
\hline In store & $41.33(37.10,45.55)$ & $58.66(54.44,62.89)$ & 0.001 & & & \\
\hline Other than store & $45.71(41.11,50.30)$ & $54.28(49.69,58.88)$ & 0.001 & & & \\
\hline Sport or promotion event (bidi) & $55.56(15.04,96.06)$ & $44.44(3.93,84.95)$ & 0.618 & & & \\
\hline Other promotions (bidi) & $33.33(27.50,39.15)$ & $66.67(60.84,72.49)$ & $<0.001$ & & & \\
\hline Any advertisement (Smokeless) & & & & $45.68(40.50,50.85)$ & $54.31(49.14,59.49)$ & 0.109 \\
\hline In store & & & & $44.72(38.81,50.64)$ & $55.27(49.35,61.18)$ & 0.578 \\
\hline Other than store & & & & $44.72(37.75,51.69)$ & $55.27(48.30,62.24)$ & 0.395 \\
\hline Sport or promotion event & & & & $33.33(0.00,100.76)$ & $66.67(0.00,200.10)$ & 0.728 \\
\hline Other promotions & & & & $36.91(26.36,47.43)$ & $60.09(52.56,73.63)$ & 0.227 \\
\hline
\end{tabular}

*Proportional value with $95 \%$ confidence Interval has been reported

${ }^{* *}$ In the last 30 days, respondent had noticed any advertisements or signs promoting events that encouraged respondent to tobacco use.

Table 4: Comparing various marketing policies to residence.

\begin{tabular}{|c|c|c|c|c|c|c|c|c|c|c|}
\hline \multirow{4}{*}{$\begin{array}{l}\text { Socio- } \\
\text { economic and } \\
\text { demographic } \\
\text { variables }\end{array}$} & \multicolumn{10}{|c|}{ Marketing policy } \\
\hline & \multicolumn{5}{|c|}{ Cigarettes } & \multicolumn{5}{|c|}{ Bidi } \\
\hline & \multicolumn{3}{|c|}{ Advertisement } & \multirow{2}{*}{$\begin{array}{l}\text { Sport or } \\
\text { promotion } \\
\text { event }\end{array}$} & \multirow{2}{*}{$\begin{array}{c}\text { Other } \\
\text { promotions }\end{array}$} & \multicolumn{3}{|c|}{ Advertisement } & \multirow{2}{*}{$\begin{array}{l}\text { Sport or } \\
\text { promoting } \\
\text { events\% } \\
(95 \% \mathrm{Cl})\end{array}$} & \multirow{2}{*}{$\begin{array}{c}\text { Other } \\
\text { promotion } \% \\
(95 \% \mathrm{Cl})\end{array}$} \\
\hline & $\begin{array}{c}\text { Anywhere\% } \\
(95 \% \mathrm{Cl})\end{array}$ & $\begin{array}{l}\text { In store } \% \\
(95 \% \mathrm{Cl})\end{array}$ & $\begin{array}{c}\text { Other than } \\
\text { store } \%(95 \% \\
\text { Cl) }\end{array}$ & & & $\begin{array}{c}\text { Anywhere\% } \\
(95 \% \mathrm{Cl})\end{array}$ & $\begin{array}{l}\text { In store } \% \\
(95 \% \mathrm{Cl})\end{array}$ & $\begin{array}{l}\text { Other than } \\
\text { store } \%(95 \% \\
\text { Cl) }\end{array}$ & & \\
\hline \multicolumn{11}{|l|}{ Residence } \\
\hline Urban & $\begin{array}{c}48.84 \\
(46.01,51.66)\end{array}$ & $\begin{array}{c}46.61 \\
(43.53,49.67)\end{array}$ & $\begin{array}{c}51.57 \\
(47.94,55.20)\end{array}$ & $\begin{array}{c}62.07 \\
(43.28,80.85)\end{array}$ & $\begin{array}{c}51.24 \\
(47.24,55.24)\end{array}$ & $\begin{array}{c}45.25 \\
(41.53,48.95)\end{array}$ & $\begin{array}{c}41.33(37.10 \\
45.55)\end{array}$ & $\begin{array}{c}45.71 \\
(41.11,50.31)\end{array}$ & $\begin{array}{c}55.56 \\
(15.04,96.06)\end{array}$ & $\begin{array}{c}33.33 \\
(27.50,39.15)\end{array}$ \\
\hline Rural & $\begin{array}{c}51.16(48.34 \\
53.99)\end{array}$ & $\begin{array}{c}53.39(50.32 \\
56.46)\end{array}$ & $\begin{array}{c}48.43(44.79 \\
52.05)\end{array}$ & $\begin{array}{c}37.93 \\
(19.15,56.72)\end{array}$ & $\begin{array}{c}48.76 \\
(44.75,52.75)\end{array}$ & $\begin{array}{c}54.75 \\
(51.04,58.46)\end{array}$ & $\begin{array}{c}58.67(54.44 \\
62.89)\end{array}$ & $\begin{array}{c}54.29(49.69 \\
58.89)\end{array}$ & $\begin{array}{c}44.44 \\
(3.93,84.95)\end{array}$ & $\begin{array}{c}66.67 \\
(60.84,72.49)\end{array}$ \\
\hline \multicolumn{11}{|l|}{ Gender } \\
\hline Male & $\begin{array}{c}98.59(97.92 \\
99.26)\end{array}$ & $\begin{array}{c}98.53(97.78 \\
99.26)\end{array}$ & $\begin{array}{c}99.32(98.72 \\
99.91)\end{array}$ & $\begin{array}{c}96.55 \\
(89.49,100.0)\end{array}$ & $\begin{array}{c}98.51 \\
(57.53,99.74)\end{array}$ & \begin{tabular}{|c|}
$97.83(96.75$ \\
$98.92)$
\end{tabular} & $\begin{array}{c}97.33(95.95 \\
98.71)\end{array}$ & $\begin{array}{c}99.12(98.26 \\
99.98)\end{array}$ & $\begin{array}{c}88.89 \\
(63.26,100.0)\end{array}$ & $\begin{array}{c}98.04 \\
(96.32,99.75)\end{array}$ \\
\hline Female & $\begin{array}{c}1.41(0.74 \\
2.07)\end{array}$ & $\begin{array}{c}1.47(0.73 \\
2.21)\end{array}$ & $\begin{array}{c}0.68 \\
(0.09,1.28)\end{array}$ & $\begin{array}{c}3.45 \\
(3.61,10.51)\end{array}$ & $\begin{array}{c}1.49 \\
(0.21,2.46)\end{array}$ & $\begin{array}{l}2.16(1.07 \\
3.24)\end{array}$ & $\begin{array}{c}2.67(1.29 \\
4.05)\end{array}$ & $\begin{array}{c}0.88(0.081 \\
1.74)\end{array}$ & $\begin{array}{c}11.11 \\
(10.51,36.73)\end{array}$ & $\begin{array}{c}1.96 \\
(0.24,3.67)\end{array}$ \\
\hline Age $(y r s)^{*}$ & $35.63(0.22)$ & $35.84(0.25)$ & $35.08(0.28)$ & $33.25(1.30)$ & $34.49(0.33)$ & $35.97(0.29)$ & $36.30(0.33)$ & $36.03(0.37)$ & $34.59(2.07)$ & $35.07(0.52)$ \\
\hline
\end{tabular}


Citation: Sultana P, Rahman MT, Roy DC (2017) Marketing Policy that Accelerate Tobacco Use in Bangladesh: A Statistical Investigation. J Biom Biostat 8: 386. doi: 10.4172/2155-6180.1000386

Page 5 of 8

\begin{tabular}{|c|c|c|c|c|c|c|c|c|c|c|}
\hline \multirow{4}{*}{$\begin{array}{l}\text { Socio- } \\
\text { economic and } \\
\text { demographic } \\
\text { variables }\end{array}$} & \multicolumn{10}{|c|}{ Marketing policy } \\
\hline & \multicolumn{5}{|c|}{ Cigarettes } & \multicolumn{5}{|c|}{ Bidi } \\
\hline & \multicolumn{3}{|c|}{ Advertisement } & \multirow{2}{*}{$\begin{array}{l}\text { Sport or } \\
\text { promotion } \\
\text { event }\end{array}$} & \multirow{2}{*}{$\begin{array}{c}\text { Other } \\
\text { promotions }\end{array}$} & \multicolumn{3}{|c|}{ Advertisement } & \multirow{2}{*}{$\begin{array}{l}\text { Sport or } \\
\text { promoting } \\
\text { events\% } \\
(95 \% \mathrm{Cl})\end{array}$} & \multirow{2}{*}{$\begin{array}{c}\text { Other } \\
\text { promotion } \% \\
(95 \% \mathrm{Cl})\end{array}$} \\
\hline & $\begin{array}{l}\text { Anywhere\% } \\
(95 \% \mathrm{Cl})\end{array}$ & $\begin{array}{c}\text { In store\% } \\
(95 \% \mathrm{Cl})\end{array}$ & $\begin{array}{c}\text { Other than } \\
\text { store } \%(95 \% \\
\text { Cl) }\end{array}$ & & & $\begin{array}{c}\text { Anywhere\% } \\
(95 \% \mathrm{Cl})\end{array}$ & \begin{tabular}{|c|} 
In store \% \\
$(95 \% \mathrm{Cl})$
\end{tabular} & $\begin{array}{c}\text { Other than } \\
\text { store\% }(95 \% \\
\mathrm{Cl})\end{array}$ & & \\
\hline \multicolumn{11}{|l|}{ Educational level } \\
\hline $\begin{array}{l}\text { No formal } \\
\text { schooling }\end{array}$ & $\begin{array}{c}42.85 \\
(40.05,45.65)\end{array}$ & $\begin{array}{c}44.93 \\
(41.87,47.99)\end{array}$ & $\begin{array}{c}38.57 \\
(35.04,42.11)\end{array}$ & $\begin{array}{c}31.03 \\
(13.12,48.94)\end{array}$ & $\begin{array}{c}42.45 \\
(38.49,46.41)\end{array}$ & $\begin{array}{c}47.98 \\
(44.25,51.70)\end{array}$ & $\begin{array}{c}51.42 \\
(47.13,55.71)\end{array}$ & $\begin{array}{c}43.51 \\
(38.94,48.08)\end{array}$ & $\begin{array}{c}33.33 \\
(5.10,71.76)\end{array}$ & $\begin{array}{c}45.88 \\
(39.72,52.03)\end{array}$ \\
\hline $\begin{array}{l}\text { Less than primary } \\
\text { school completed }\end{array}$ & $\begin{array}{c}18.52(16.32 \\
20.71)\end{array}$ & $\begin{array}{c}16.81 \\
(14.51,19.11)\end{array}$ & $\begin{array}{c}19.42 \\
(16.55,22.30)\end{array}$ & $\begin{array}{c}17.24 \\
(2.61,31.86)\end{array}$ & $\begin{array}{c}17.91(14.84 \\
20.97)\end{array}$ & $\begin{array}{c}17.43 \\
(14.60,20.26)\end{array}$ & $\begin{array}{c}16.19 \\
(13.02,19.35)\end{array}$ & $\begin{array}{c}19.34 \\
(1569,22.98)\end{array}$ & $\begin{array}{c}22.22(11.67 \\
56.11)\end{array}$ & $\begin{array}{c}15.68 \\
(11.19,20.18)\end{array}$ \\
\hline $\begin{array}{l}\text { Primary School } \\
\text { Completed }\end{array}$ & $\begin{array}{c}8.97(7.35 \\
10.58)\end{array}$ & $\begin{array}{c}9.34 \\
(7.54,11.13)\end{array}$ & $\begin{array}{c}8.75 \\
(6.70,10.80)\end{array}$ & $\begin{array}{c}17.24(2.61 \\
31.86)\end{array}$ & $\begin{array}{c}10.94 \\
(8.44,13.44)\end{array}$ & $\begin{array}{c}8.78 \\
(6.67,10.90)\end{array}$ & $\begin{array}{c}8.76 \\
(6.33,11.18)\end{array}$ & $\begin{array}{c}8.57 \\
(5.98,11.15)\end{array}$ & $\begin{array}{c}11.11 \\
(10.11,36.73)\end{array}$ & $\begin{array}{c}12.15 \\
(8.11,16.19)\end{array}$ \\
\hline $\begin{array}{l}\text { Less than } \\
\text { secondary School } \\
\text { completed }\end{array}$ & $\begin{array}{c}17.10 \\
(14.97,19.23)\end{array}$ & $\begin{array}{c}16.91 \\
(14.60,19.22)\end{array}$ & $\begin{array}{c}18.60 \\
(15.77,21.43)\end{array}$ & $\begin{array}{c}10.34(1.44 \\
22.13)\end{array}$ & $\begin{array}{c}17.74 \\
(14.68,20.80)\end{array}$ & $\begin{array}{c}15.56 \\
(12.85,18.26)\end{array}$ & $\begin{array}{c}13.90 \\
(10.93,16.87)\end{array}$ & $\begin{array}{c}17.80 \\
(14.27,21.33)\end{array}$ & -- & $\begin{array}{c}17.64(12.93 \\
22.35)\end{array}$ \\
\hline $\begin{array}{l}\text { Secondary school } \\
\text { completed }\end{array}$ & $\begin{array}{l}5.89(4.56 \\
\quad 7.22)\end{array}$ & $\begin{array}{c}5.50 \\
(4.10,6.91)\end{array}$ & $\begin{array}{c}6.83 \\
(5.00,8.67)\end{array}$ & $\begin{array}{c}3.44 \\
(3.61,10.51)\end{array}$ & $\begin{array}{c}5.63 \\
(3.79,7.48)\end{array}$ & $\begin{array}{c}3.89 \\
(2.44,5.33)\end{array}$ & $\begin{array}{c}3.23 \\
(1.71,4.75)\end{array}$ & $\begin{array}{c}4.39 \\
(2.50,6.28)\end{array}$ & -- & $\begin{array}{c}5.09 \\
(2.38,7.81)\end{array}$ \\
\hline $\begin{array}{l}\text { High school } \\
\text { completed }\end{array}$ & $\begin{array}{l}2.49(1.60 \\
3.37)\end{array}$ & $\begin{array}{c}2.26 \\
(1.34,3.17)\end{array}$ & $\begin{array}{c}2.87 \\
(1.65,4.08)\end{array}$ & $\begin{array}{c}3.44 \\
(3.61,10.51)\end{array}$ & $\begin{array}{c}1.82 \\
(.75,2.89)\end{array}$ & $\begin{array}{c}2.44 \\
(1.29,3.60)\end{array}$ & $\begin{array}{c}2.47 \\
(1.14,3.80)\end{array}$ & $\begin{array}{c}1.97 \\
(0.69,3.26)\end{array}$ & -- & $\begin{array}{c}1.56 \\
(0.03,3.10)\end{array}$ \\
\hline $\begin{array}{l}\text { College/University } \\
\text { Completed and/ } \\
\text { higher }\end{array}$ & $\begin{array}{l}4.15(3.02 \\
5.28)\end{array}$ & $\begin{array}{c}4.22 \\
(2.98,5.46)\end{array}$ & $\begin{array}{c}4.92 \\
(3.35,6.49)\end{array}$ & $\begin{array}{c}17.24 \\
(2.61,31.86)\end{array}$ & $\begin{array}{c}3.48 \\
(2.01,4.95)\end{array}$ & $\begin{array}{c}3.89 \\
(2.44,5.33)\end{array}$ & $\begin{array}{c}4.00 \\
(2.31,5.68)\end{array}$ & $\begin{array}{c}4.39 \\
(2.50,6.28)\end{array}$ & $\begin{array}{c}33.33 \\
(5.10,71.76)\end{array}$ & $\begin{array}{c}1.96 \\
(0.24,3.67)\end{array}$ \\
\hline \multicolumn{11}{|l|}{ Occupation } \\
\hline $\begin{array}{l}\text { Employment } \\
\text { (Government, } \\
\text { Non-Government) }\end{array}$ & \begin{tabular}{|c|}
$12.04(10.20$ \\
$13.88)$
\end{tabular} & $\begin{array}{c}11.30 \\
(9.35,13.25)\end{array}$ & $\begin{array}{c}13.95 \\
(11.43,16.47)\end{array}$ & $\begin{array}{c}17.24 \\
(2.61,31.86)\end{array}$ & $\begin{array}{c}10.44 \\
(7.99,12.89)\end{array}$ & $\begin{array}{c}10.08 \\
(7.84,12.33)\end{array}$ & $\begin{array}{c}9.33 \\
(6.83,11.82)\end{array}$ & $\begin{array}{c}11.20 \\
(8.29,14.11)\end{array}$ & $\begin{array}{c}11.11(10.51 \\
36.73)\end{array}$ & $\begin{array}{c}6.27 \\
(3.27,9.27)\end{array}$ \\
\hline $\begin{array}{l}\text { Business (small, } \\
\text { large) }\end{array}$ & $\begin{array}{c}23.17(20.78 \\
25.55)\end{array}$ & $\begin{array}{c}23.20 \\
(20.60,25.80)\end{array}$ & $\begin{array}{c}23.80 \\
(20.70,26.89)\end{array}$ & $\begin{array}{c}13.79(0.44 \\
27.14)\end{array}$ & $\begin{array}{c}25.37 \\
(21.89,28.85)\end{array}$ & $\begin{array}{c}21.46 \\
(18.40,24.53)\end{array}$ & $\begin{array}{c}21.14 \\
(17.63,24.64)\end{array}$ & $\begin{array}{c}21.31 \\
(17.54,25.09)\end{array}$ & $\begin{array}{c}11.11(10.51 \\
36.73)\end{array}$ & $\begin{array}{c}26.27 \\
(20.83,31.71)\end{array}$ \\
\hline $\begin{array}{l}\text { Farming (land } \\
\text { owner and } \\
\text { farmer) }\end{array}$ & $\begin{array}{c}18.18(16.00 \\
20.37)\end{array}$ & $\begin{array}{c}18.87 \\
(16.46,21.28)\end{array}$ & $\begin{array}{c}17.51 \\
(14.74,20.27)\end{array}$ & $\begin{array}{c}31.03(13.12 \\
48.94)\end{array}$ & $\begin{array}{c}18.24 \\
(15.15,21.33)\end{array}$ & $\begin{array}{c}19.45 \\
(16.50,22.40)\end{array}$ & $\begin{array}{c}20.95 \\
(17.45,24.44)\end{array}$ & $\begin{array}{c}20.00 \\
(16.31,23.68)\end{array}$ & $\begin{array}{c}33.33 \\
(5.10,71.76)\end{array}$ & $\begin{array}{c}23.52 \\
(18.28,28.77)\end{array}$ \\
\hline $\begin{array}{l}\text { Agricultural/ } \\
\text { Industrial worker/ } \\
\text { daily laborer/ } \\
\text { Other self- } \\
\text { employed }\end{array}$ & $\begin{array}{c}35.04(32.35 \\
37.74)\end{array}$ & $\begin{array}{c}35.49 \\
(32.55,38.44)\end{array}$ & $\begin{array}{c}34.47 \\
(30.01,37.92)\end{array}$ & $\begin{array}{c}20.68 \\
(5.00,36.37)\end{array}$ & $\begin{array}{c}33.00 \\
(29.23,36.76)\end{array}$ & $\begin{array}{c}37.89 \\
(34.27,41.51)\end{array}$ & $\begin{array}{c}38.85 \\
(34.67,43.04)\end{array}$ & $\begin{array}{c}37.36 \\
(32.90,41.82)\end{array}$ & $\begin{array}{c}22.22 \\
(11.67,56.11)\end{array}$ & $\begin{array}{c}32.15 \\
(26.38,37.92)\end{array}$ \\
\hline $\begin{array}{l}\text { Homemaker/ } \\
\text { Housework }\end{array}$ & $\begin{array}{c}0.91(0.37 \\
1.45)\end{array}$ & $\begin{array}{c}0.98 \\
(0.37,1.59)\end{array}$ & $\begin{array}{c}0.27 \\
(0.10,0.65)\end{array}$ & -- & $\begin{array}{c}0.82 \\
(0.10,1,55)\end{array}$ & $\begin{array}{c}1.29 \\
(0.45,2.14)\end{array}$ & $\begin{array}{c}1.71 \\
(0.60,2.82)\end{array}$ & $\begin{array}{c}0.22 \\
(0.21,0.65,)\end{array}$ & -- & $\begin{array}{c}1.17 \\
(0.15,2.50)\end{array}$ \\
\hline $\begin{array}{l}\text { Retired and } \\
\text { unemployed (able } \\
\text { to work/unable to } \\
\text { work) }\end{array}$ & $\begin{array}{c}3.32(2.30 \\
4.33)\end{array}$ & $\begin{array}{c}2.55 \\
(1.58,3.52)\end{array}$ & $\begin{array}{c}3,55 \\
(2,21,4.90)\end{array}$ & $\begin{array}{c}6.89 \\
(2.91,16.70)\end{array}$ & $\begin{array}{c}3.31 \\
(1.88,4.75)\end{array}$ & $\begin{array}{c}2.73 \\
(1.52,3.95)\end{array}$ & $\begin{array}{c}1.52 \\
(0.47,2.57)\end{array}$ & $\begin{array}{c}3.07 \\
(1.48,4.66)\end{array}$ & $\begin{array}{c}11.11(10.51 \\
36.73)\end{array}$ & $\begin{array}{c}2.35 \\
(4.79,4.22)\end{array}$ \\
\hline Student/Other & $\begin{array}{c}7.30(5.83 \\
8.78)\end{array}$ & $\begin{array}{c}7.57 \\
(5.94,9.19)\end{array}$ & $\begin{array}{c}6.42 \\
(4.64,8.21)\end{array}$ & $\begin{array}{c}10.34(1.44 \\
22.13)\end{array}$ & $\begin{array}{c}8.78 \\
(6.52,11.05)\end{array}$ & $\begin{array}{c}7.06 \\
(5.14,8.97)\end{array}$ & $\begin{array}{c}6.47 \\
(4.36,8.58)\end{array}$ & $\begin{array}{l}6.81(4.48 \\
9.13)\end{array}$ & $\begin{array}{c}11.11(10.51 \\
36.73)\end{array}$ & $\begin{array}{c}8.23 \\
(4.83,11.63)\end{array}$ \\
\hline \multicolumn{11}{|l|}{ Wealth index } \\
\hline Lowest & $\begin{array}{c}20.51(18.23 \\
22.79)\end{array}$ & $\begin{array}{c}20.84 \\
(18.34,23.34)\end{array}$ & $\begin{array}{c}19.15 \\
(16.29,22.01)\end{array}$ & $\begin{array}{c}10.34 \\
(1.44,22.13)\end{array}$ & $\begin{array}{c}20.89 \\
(17.64,24.14)\end{array}$ & $\begin{array}{c}21.61 \\
(18.54,24.68)\end{array}$ & $\begin{array}{c}21.33 \\
(17.81,24.84)\end{array}$ & $\begin{array}{c}21.31 \\
(17.54,25.09)\end{array}$ & $\begin{array}{c}11.11(10.51 \\
36.73)\end{array}$ & $\begin{array}{c}20.78(15.77 \\
25.89)\end{array}$ \\
\hline Low & $\begin{array}{c}26.91(24.40 \\
29.41)\end{array}$ & $\begin{array}{c}26.84 \\
(24.11,29.57)\end{array}$ & $\begin{array}{c}25.58 \\
(22.41,28.75)\end{array}$ & $\begin{array}{c}31.03 \\
(13.12,48.94)\end{array}$ & $\begin{array}{c}24.87 \\
(21.41,28.33)\end{array}$ & $\begin{array}{c}28.38 \\
(25.02,31.74)\end{array}$ & $\begin{array}{c}28.95 \\
(25.05,32.84)\end{array}$ & $\begin{array}{c}26.15 \\
(22.10,3020)\end{array}$ & $\begin{array}{c}11.11(10.51 \\
36.73)\end{array}$ & $\begin{array}{c}25.49 \\
(20.10,30.87)\end{array}$ \\
\hline Middle & $\begin{array}{c}18.43(16.24 \\
20.63)\end{array}$ & $\begin{array}{c}19.17 \\
(16.75,21.59)\end{array}$ & $\begin{array}{c}18.60 \\
(15.77,21.43)\end{array}$ & $\begin{array}{c}13.79 \\
(0.44,27.14)\end{array}$ & $\begin{array}{c}19.73 \\
(16.54,22.92)\end{array}$ & $\begin{array}{c}18.01 \\
(15.14,20.87)\end{array}$ & $\begin{array}{c}18.47(15.14 \\
21.80)\end{array}$ & $\begin{array}{c}19.34 \\
(15.69,22.98)\end{array}$ & $\begin{array}{c}22.22 \\
(11.67,56.11)\end{array}$ & $\begin{array}{c}24.31 \\
(19.01,29.61)\end{array}$ \\
\hline High & $\begin{array}{c}20.34(18.07 \\
22.62)\end{array}$ & $\begin{array}{c}19.56 \\
(17.12,22.00)\end{array}$ & $\begin{array}{c}20.79 \\
(17.84,23.74)\end{array}$ & $\begin{array}{c}20.68 \\
(5.00,36.37)\end{array}$ & $\begin{array}{c}21.22 \\
(17.95,24.50)\end{array}$ & $\begin{array}{c}19.74 \\
(16.77,22.70)\end{array}$ & $\begin{array}{c}20.19 \\
(16.74,23.63)\end{array}$ & $\begin{array}{c}19.78(16.10 \\
34.54)\end{array}$ & $\begin{array}{c}11.11(10.51 \\
36.73)\end{array}$ & $\begin{array}{c}21.17 \\
(16.12,26.22)\end{array}$ \\
\hline Highest & $\begin{array}{c}13.78 \\
(11.83,15.73)\end{array}$ & $\begin{array}{c}13.56 \\
(11.46,15.67)\end{array}$ & $\begin{array}{c}15.86 \\
(13.21,18.52)\end{array}$ & $\begin{array}{c}24.13 \\
(7.57,40.70)\end{array}$ & $\begin{array}{c}17.26 \\
(10.55,15.98)\end{array}$ & $\begin{array}{c}22.24 \\
(9.80,14.69)\end{array}$ & $\begin{array}{c}11.04 \\
(8.35,13.73)\end{array}$ & $\begin{array}{c}13.40(10.64 \\
16.54)\end{array}$ & $\begin{array}{c}44.44 \\
(3.93,84.95)\end{array}$ & $\begin{array}{c}8.23 \\
(4.83,11.63)\end{array}$ \\
\hline
\end{tabular}

*Mean with SD is reported.

-- No observation found.

${ }^{* *}$ In the last 30 days, respondent had noticed any advertisements or signs promoting events that encouraged respondent to tobacco use.

Table 5: Comparing various cofactors to marketing policy (smoking tbacco products).

$\mathrm{CI}=1.28,2.11)$ are more likely to be inspired by the marketing policy to smokeless tobacco use in preceding 30 days. Respondents of middle wealth index is more likely to be inspired $(\mathrm{OR}=1.21,95 \% \mathrm{CI}=1.01$, 1.46) by the marketing policy to use smokeless tobacco product than respondents of lowest wealth index. On the other hand the odds ratio for respondents of highest wealth index is $\mathrm{OR}=0.89,95 \% \mathrm{CI}=0.70,1.13$ implying that 0.89 times less likely to be inspired by the marketing policy to use smokeless tobacco product than respondents of lowest wealth index. 
Citation: Sultana P, Rahman MT, Roy DC (2017) Marketing Policy that Accelerate Tobacco Use in Bangladesh: A Statistical Investigation. J Biom Biostat 8: 386. doi: 10.4172/2155-6180.1000386

Page 6 of 8

\begin{tabular}{|c|c|c|c|c|c|}
\hline \multirow[t]{3}{*}{ Socio-economic and demographic variables } & \multicolumn{5}{|c|}{ Marketing policy** } \\
\hline & \multicolumn{3}{|c|}{ Advertisement } & \multirow{2}{*}{$\begin{array}{l}\text { Sport or promoting } \\
\text { event } \%(95 \% \mathrm{Cl})\end{array}$} & \multirow{2}{*}{$\begin{array}{l}\text { Other promotion } \% \\
(95 \% \mathrm{Cl})\end{array}$} \\
\hline & Anywhere\% $(95 \% \mathrm{Cl})$ & In store\% $(95 \% \mathrm{Cl})$ & $\begin{array}{l}\text { Other than store } \% \\
\qquad(95 \% \mathrm{Cl})\end{array}$ & & \\
\hline \multicolumn{6}{|l|}{ Residence } \\
\hline Urban & $45.69(40.50,50.85)$ & $44.73(38.8150 .64)$ & $44.72(37.75,51.69)$ & $33.33(0.00,100.76)$ & $36.91(26.36,47.43)$ \\
\hline Rural & $54.31(49.14,59.49)$ & $55.27(49.35,61.18)$ & $55.28(48.31,62.24)$ & $66.67(0.00,200.10)$ & $60.09(52.56,73.63)$ \\
\hline \multicolumn{6}{|l|}{ Gender } \\
\hline Male & $55.15(49.9860 .32)$ & $49.82(43.87,55.76)$ & $69.34(62.88,75.80)$ & $66.67(0.00,200.10)$ & $67.85(57.66,78.06)$ \\
\hline Female & $44.84(39.67,50.01)$ & $50.18(44.23,56.12)$ & $30.65(24.19,37.11)$ & $33.33(0.00,100.76)$ & $32.14(21.94,42.34)$ \\
\hline Age $(y r s)^{*}$ & $36.41(0.38)$ & $36.57(0.45)$ & $36.77(0.52)$ & $30.80(2.19)$ & $35.85(0.81)$ \\
\hline \multicolumn{6}{|l|}{ Educational level } \\
\hline No formal schooling & $52.64(47.45,57.83)$ & $54.91(48.99,60.83)$ & $46.73(39.74,53.72)$ & $66.67(0.00,200.10)$ & $46.42(35.54,57.31)$ \\
\hline Less than primary school completed & $16.71(12.83,20.59)$ & $16.00(11.63,20.36)$ & $17.08(11.81,22.36)$ & -- & $16.67(8.53,24.80)$ \\
\hline Primary School Completed & $10.58(7.38,13.78)$ & $9.45(5.97,12.93)$ & $12.56(7.91,12.20)$ & $33.33(0.00,100.76)$ & $13.09(5.73,20.46)$ \\
\hline Less than secondary school completed & $11.97(8.60,15.35)$ & 10.54 (6.89. 14.19) & $13.56(8.76,18.36)$ & -- & $13.09(5.73,20.46)$ \\
\hline Secondary school completed & $3.34(1.47,5.21)$ & $4.00(1.66,6.33)$ & $5.52(2.32,8.73)$ & -- & $4.76(0.11,9.411)$ \\
\hline High school completed & $3.06(1.27,4.85)$ & $3.63(1.41,5.86)$ & $2.51(0.31,4.70)$ & -- & $3.57(0.00,7,62)$ \\
\hline College/University Completed and/higher & $1.67(0.33,3.00)$ & $1.45(0.03,2.87)$ & $2.01(0.04,3.97)$ & -- & $2.38(0.00,5.70)$ \\
\hline \multicolumn{6}{|l|}{ Occupation } \\
\hline Employment (Government, Non-Government) & $8.35(5.48,11.23)$ & $6.18(3.31,9.04)$ & $11.55(7.07,16.03)$ & $33.33(0.00,100.76)$ & $5.95(7.86,11.11)$ \\
\hline Business (small, large) & $12.53(9.09,15.97)$ & $10.90(7.20,14.61)$ & $15.07(10.06,20.08)$ & -- & $10.71(3.96,17.46)$ \\
\hline Farming (land owner and farmer) & $12.53(9.09,15.97)$ & $12.36(8.44,16.27)$ & $14.57(9.62,19.51)$ & -- & $21.42(12.47,30.38)$ \\
\hline $\begin{array}{l}\text { Agricultural/Industrial worker/daily laborer/Other } \\
\text { self- employed }\end{array}$ & $25.06(20.56,29.57)$ & $24.36(19.25,29.46)$ & $28.14(21.83,34.44)$ & $33.33(0.00,100.76)$ & $23.80(14.51,33.10)$ \\
\hline Homemaker/Housework & $32.59(27.71,37.46)$ & $37.45(31.69,43.21)$ & $21.60(15.84,27.37)$ & $33.33(0.00,100.76)$ & $23.80(14.51,33.10)$ \\
\hline $\begin{array}{l}\text { Retired and unemployed (able to work/unable } \\
\text { to work) }\end{array}$ & $3.89(1.88,5.91)$ & $3.63(1.41,5.86)$ & $5.02(1.96,8.08)$ & -- & $35.71(0.00,7.62)$ \\
\hline Student/Other & $5.01(2.74,7.28)$ & $5.09(2.47,7.70)$ & $4.02(1.26,6.77)$ & -- & $10.71(3.96,17.46)$ \\
\hline \multicolumn{6}{|l|}{ Wealth index } \\
\hline Lowest & $26.18(21.61,30.75)$ & $25.81(20.61,31.02)$ & $21.10(15.38,25.62)$ & $33.33(0.00,100.76)$ & $16.67(8.53,24.80)$ \\
\hline Low & $25.62(21.08,30.16)$ & $25.09(19.93,30.24)$ & $24.12(18.12,30.11)$ & $33.33(0.00,100.76)$ & $21.42(12.47,30.38)$ \\
\hline Middle & $20.05(15.89,24.21)$ & $20.72(15.90,25.54)$ & $23.11(17.20,29.02)$ & -- & $32.14(21.94,42.33)$ \\
\hline High & $18.10(14.10,22.10)$ & $18.18(13.59,22.76)$ & $20.60(14.93,26.27)$ & $33.33(0.00 .10,100.76)$ & $15.47(7.58,23.37)$ \\
\hline Highest & $10.02(6.90,13.14)$ & $10.18(6.58,13.77)$ & $11.05(6.66,15.44)$ & -- & $14.28(6.64,21.92)$ \\
\hline
\end{tabular}

*Mean with SD is reported, -- No observation found, ${ }^{* *}$ In the last 30 days, have been noticed any advertisements or signs promoting tobacco products that encouraged respondent to tobacco use.

Table 6: Comparing various cofactors to marketing policy (smokeless tobacco products).

\section{Discussion}

This study is the first study that has taken into considers the active marketing policies that are working as a burden in reduction of tobacco use in Bangladesh based on a nationwide survey. This study found that most of the successful marketing policies that inspired the user were found in store for both smoking (Bidi/Cigarettes) and smokeless tobacco products. Tobacco users are more likely to be inspired in preceding 30 days by marketing policy than non-user. But a big part of non-user was inspired, too. It is alarming for the society. It has been found that besides male, a big part of female respondent was inspired to tobacco use by the marketing policy which is not acceptable. Social norm of Bangladesh does not allow female to tobacco use, except zarda and sadapata [20,21]. Younger respondents were more inspired than older respondents. It is also alarming for the society. It indicates that such marketing policy is recruiting more onset of tobacco user. Higher educated peoples were more likely to be inspired to tobacco use in the preceding 30 days. It is not expectable; education usually found to play an important role for global and public health problems $[22,23]$. Students were most likely to be inspired by marketing policy to tobacco smoking. On the other hand, peoples involved in farming were most likely to be inspired by marketing policy to use smokeless tobacco product. Peoples of medium socio-economic status are more likely to be inspired by marketing policy. However, such pattern is difficult interpret.

\section{Strength and Limitation}

The major strengths of this study include large sample size based on nationwide survey, the coverage of men and women respondents, and coverage of both rural and urban areas. However, there are several limitations which need to be addressed. The findings in this report are based on self-reports. Furthermore, education categories were combined into broad groupings, which could have contributed to biased estimates in terms of the gradients observed. Nonetheless, these groupings provided greater precision than those used in earlier tobacco related research in Bangladesh. Respondents according to job categories were too much skewed and were regrouped. For the regrouping, no proper reference in literature was found. Therefore, regrouping was based on personal justification based on countryside. The data used in constructing wealth index was based on limited number of asset variables, which might result in incomplete or under representing socioeconomic status. Some other variables like psychological or medical conditions related variables could provide more predicting accuracy, but such variables were not available. 
Citation: Sultana P, Rahman MT, Roy DC (2017) Marketing Policy that Accelerate Tobacco Use in Bangladesh: A Statistical Investigation. J Biom Biostat 8: 386. doi: 10.4172/2155-6180.1000386

Page 7 of 8

\begin{tabular}{|c|c|c|}
\hline Socio- demographic and economic variables & $\begin{array}{l}\text { Marketing policy (smoking tobacco } \\
\text { product) OR }(95 \% \mathrm{Cl})\end{array}$ & $\begin{array}{l}\text { Marketing policy (smokeless tobacco product) } \\
\text { OR }(95 \% \mathrm{Cl})\end{array}$ \\
\hline Tobacco use & $1.25(1.10,1.42)$ & $1.31(1.14,1.51)$ \\
\hline \multicolumn{3}{|l|}{ Residence } \\
\hline Urban (RC) & 1.00 & 1.00 \\
\hline Rural & $1.17(1.06,1.30)$ & $1.15(1.02,1.31)$ \\
\hline \multicolumn{3}{|l|}{ Gender } \\
\hline Male (RC) & 1.00 & 1.00 \\
\hline Female & $0.24(0.20,0.28)$ & $0.63(0.51,0.77)$ \\
\hline Age & $0.98(0.98,0.99)$ & $0.99(0.98,0.99)$ \\
\hline \multicolumn{3}{|l|}{ Education } \\
\hline No formal schooling (RC) & 1.00 & 1.00 \\
\hline Less than primary school completed & $0.97(0.85,1.12)$ & $0.83(0.69,1.00)$ \\
\hline Primary School Completed & $1.25(1.07,1.46)$ & $1.08(0.89,1.32)$ \\
\hline Less than secondary school completed & $1.24(1.08,1.43)$ & $1.06(0.89,1.28)$ \\
\hline Secondary school completed & $1.42(1.15,1.74)$ & $1.21(0.93,1.57)$ \\
\hline High school completed & $1.49(1.17,1.90)$ & $1.53(1.14,2.05)$ \\
\hline College/University Completed and/higher & $1.65(1.28,2.11)$ & $1.47(1.07,2.00)$ \\
\hline \multicolumn{3}{|l|}{ Occupation } \\
\hline Employment (Government, Non-Government) (RC) & 1.00 & 1.00 \\
\hline Business (small, large) & $1.55(1.27,1.90)$ & $1.21(0.95,1.55)$ \\
\hline Farming (land owner and farmer) & $1.15(0.93,1.44)$ & $1.29(0.98,1.69)$ \\
\hline Agricultural/Industrial worker/daily laborer/Other self- employed & $1.44(1.19,1.76)$ & $1.21(0.95,1.55)$ \\
\hline Homemaker/Housework & $1.04(0.85,1.27)$ & $1.01(0.77,1.32)$ \\
\hline Retired and unemployed (able to work/unable to work) & $0.89(0.68,1.15)$ & $0.96(0.68,1.37)$ \\
\hline Student/Other & $1.61(1.30,2.00)$ & $1.16(0.89,1.52)$ \\
\hline \multicolumn{3}{|l|}{ Wealth index } \\
\hline Lowest (RC) & 1.00 & 1.00 \\
\hline Low & $1.09(0.94,1.26)$ & $1.07(0.89,1.29)$ \\
\hline Middle & $1.12(0.96,1.31)$ & $1.21(1.00,1.46)$ \\
\hline High & $1.18(1.02,1.38)$ & $1.13(0.93,1.38)$ \\
\hline Highest & $0.99(0.83,1.19)$ & $0.89(0.70,1.13)$ \\
\hline P-value from Hosmer-Lemeshow goodness of fit test & 0.8394 & 0.6662 \\
\hline AUC & 0.7342 & 0.6031 \\
\hline
\end{tabular}

RC: Reference Category; AUC: Area under the ROC Curve.

Table 7: Identifying significant correlates of being encouraged by marketing policy that accelerate tobacco use in Bangladesh using binary logistic regression.

\section{Conclusion}

The study reveals that a big proportion of non-user and female become inspired by marketing advertisement and sponsorship events. Younger, higher educated and students are significantly more inspired by such marketing advertisement and sponsorship events. Therefore, Government, policy makers and research institutions who are working for tobacco use reduction should pay attention more on these groups of people to advocate more. It has been also found that highest prevalence of marketing advertisement was found in selling store. Therefore, Government could take action to limit advertisement in selling store.

\section{Acknowledgement} Group.

The authors would like to acknowledge the members of the GATS Collaborative

\section{References}

1. World Health Organization (2008) WHO report on the global tobacco epidemic 2008: the MPOWER package. Geneva: World Health Organization.

2. Peto R, Lopez AD, Boreham J, Thun M, Health C, et al. (1996) Mortality from smoking worldwide. British Medical Bulletin 52: 12-21.

3. Sultana P, Akter S, Rahman MM, Alam MS (2015) Prevalence and Predictors of Current Tobacco Smoking in Bangladesh. J Biostatistics and Biometrics App 1: 102.

4. Siahpush M, McNeill A, Hammond D, Fong GT (2006) Socioeconomic and country variations in knowledge of health risks of tobacco smoking and toxic constituents of smoke: Results from the 2002 International Tobacco Control (ITC) Four Country Survey. Tob Control 15: iii65-iii70.

5. Wu F, Chen Y, Parvez F, Segers S, Argos M, et al. (2013) A Prospective Study of Tobacco Smoking and Mortality in Bangladesh. PLOS ONE 8: e58516.

6. Choudhury K, Hanifi SMA, Mahmood SS, Bhuiya A (2007) Sociodemographic Characteristics of Tobacco Consumers in a Rural Area of Bangladesh. Journal of Health, Population and Nutrition 25: 456-464.

7. Begum M, Sultana P (2017) Current Pattern of Product Specific Smokeless Tobacco Use in Bangladesh. J Biom Biostat 8: 362.

8. Khan MH, Khan A, Kraemer A, Mori M (2009) Prevalence and correlates of smoking among urban adult men in Bangladesh: slum versus non-slum comparison. BMC Public Health 9: 149.

9. Kabir MA, Goh KL, Kamal SM, Khan MM (2013) Tobacco smoking and its association with illicit drug use among young men aged15-24 years living in urban slums of Bangladesh. PLoS One 9: e91618.

10. Kabir MA, Goh KL, Khan MM (2013) Adolescent Tobacco Use and Its Determinants: Evidence From Global Youth Tobacco Survey, Bangladesh 2007. Asia Pac J Public Health 27: NP1578-NP1590.

11. Kabir MA, Goh KL, Khan MM (2013) Tobacco consumption and illegal drug use among Bangladeshi males: association and determinants. Am J Mens Health 7: 128-37.

12. Palupadi KM, Sinha DN, Choudhuri S, Mustafa Z, Andes L, et al. (2010) Exposure to tobacco smoke among adults in Bangladesh. Indian Journal of Public Health 55: 210-219. 
Citation: Sultana P, Rahman MT, Roy DC (2017) Marketing Policy that Accelerate Tobacco Use in Bangladesh: A Statistical Investigation. J Biom Biostat 8: 386. doi: 10.4172/2155-6180.1000386

Page 8 of 8

13. Nargis N, Ruthbah UH, Hussain AK, Fong GT, Huq I, et al. (2014) The price sensitivity of cigarette consumption in Bangladesh: evidence from the International Tobacco Control (ITC) Bangladesh Wave 1 (2009) and Wave 2 (2010) Surveys. Tob Control 23 Suppl 1: i39-47.

14. Barkat A, Chowdhury AU, Nargis N, Rahman M, Khan MS, et al. (2012) The Economics of Tobacco and Tobacco Taxation Bangladesh. International Union against Tuberculosis and Lung Disease, pp: 1-53.

15. Rahman MT, Roy DC, Sultana P (2016) Knowledge and attitude towards tobacco use in Bangladesh. Journal of Bangladesh Studies 38: 69-84.

16. Giovino GA, Mirza SA, Samet JM, Gupta PC, Jarvis MJ (2012) Tobacco use in 3 billion individuals from 16 countries: an analysis of nationally representative cross-sectional household surveys. Lancet 380: 668-679.

17. Kalsbeek WD, Bowling JM, Hsia J, Mirza S, Palipudi KM, et al. (2010) The Global Adult Tobacco Survey: sample design and related methods. Section on Survey Research Methods, pp: 1-15.
18. Rao CR (2002) Linear statistical inference and its application (2ndedn), John Wiley and Sons.

19. Christensen R (1997) Log-linear models and logistic regression. Probability Theory and Stochastic Processes Second edition. Springer, New York.

20. Sinha DN, Gupta PC, Ray CS, Singh PK (2012) Prevalence of smokeless tobacco use among adults in WHO South-East Asia. Indian Journal of Cancer 49: 342-346.

21. Huhtasaari F, Lundberg V, Eliasson M, Janlert U, Asplund K (1999) Smokeless tobacco as a possible risk factor for myocardial infarction: a population-based study in middle-aged men. Journal of the American College of Cardiology 34: 1784-1790.

22. Rahman M, Islam A, Islam M (2008) Determinants of Knowledge and Awareness about AIDS: Urban -Rural Differentials in Bangladesh. The Int J Health 9: 46-52.

23. Uddin MJ, Choudhury MN (2008) Reproductive Health Awareness among Adolescent Girls in Rural Bangladesh. Asia Pac J Public Health 20: 117-128. 